\title{
A higher order system of some coupled nonlinear Schrödinger and Korteweg-de Vries equations
}

P. Álvarez-Caudevilla, Eduardo Colorado, and Rasiel Fabelo

Citation: Journal of Mathematical Physics 58, 111503 (2017);

View online: https://doi.org/10.1063/1.5010682

View Table of Contents: http://aip.scitation.org/toc/jmp/58/11

Published by the American Institute of Physics

\section{Articles you may be interested in}

Extended symmetry analysis of generalized Burgers equations

Journal of Mathematical Physics 58, 101501 (2017); 10.1063/1.5004134

On the finite $\mathrm{W}$-algebra for the Lie superalgebra $\mathrm{Q}(\mathrm{N})$ in the non-regular case

Journal of Mathematical Physics 58, 111701 (2017); 10.1063/1.4993709

Existence and asymptotic profiles of positive solutions of quasilinear Schrödinger equations in $R^{3}$ Journal of Mathematical Physics 58, 111502 (2017); 10.1063/1.5010930

Persistence property in a two-species chemotaxis system with two signals Journal of Mathematical Physics 58, 111501 (2017); 10.1063/1.5010681

$Z_{2} \times Z_{2}$ generalizations of $\mathcal{N}=2$ super Schrödinger algebras and their representations Journal of Mathematical Physics 58, 113501 (2017); 10.1063/1.4986570

Erratum and Addendum: "Lie symmetries and group classification of a class of time fractional evolution systems" [J. Math. Phys. 56, 123504 (2015)]

Journal of Mathematical Physics 58, 119901 (2017); 10.1063/1.4992121

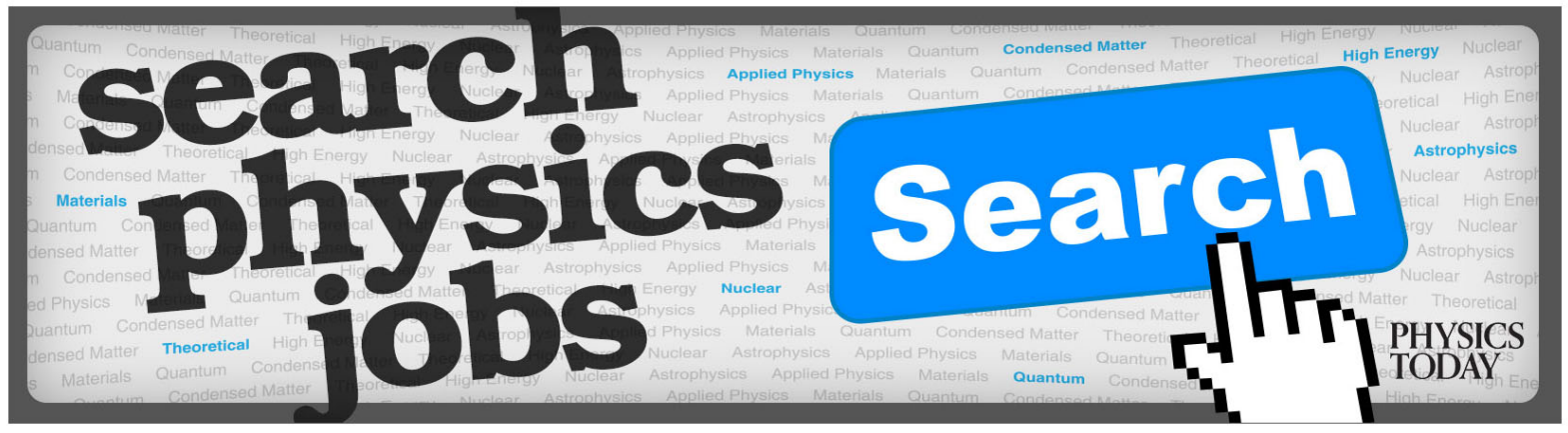




\title{
A higher order system of some coupled nonlinear Schrödinger and Korteweg-de Vries equations
}

\author{
P. Álvarez-Caudevilla, ${ }^{1,2, a)}$ Eduardo Colorado, ${ }^{1,2, b)}$ and Rasiel Fabelo ${ }^{1, c)}$ \\ ${ }^{1}$ Universidad Carlos III de Madrid, Av. Universidad 30, 28911 Leganés, Spain \\ ${ }^{2}$ Instituto de Ciencias Matemáticas, ICMAT (CSIC-UAM-UC3M-UCM), C/Nicolás Cabrera \\ 15, 28049 Madrid, Spain
}

(Received 27 February 2017; accepted 24 October 2017; published online 14 November 2017)

\begin{abstract}
We prove the existence and multiplicity of bound and ground state solutions, under appropriate conditions on the parameters, for a bi-harmonic stationary system coming from a system of coupled nonlinear Schrödinger-Korteweg-de Vries equations. We arrive at that stationary system looking for "standing-traveling" wave solutions. We first show the existence of a semi-trivial solution of the form $\left(0, V_{2}\right)$, where $V_{2}$ is a ground state of $\Delta^{2} v+\lambda_{2} v=\frac{1}{2}|v| v$. This semi-trivial solution will have the lowest energy among all the semi-trivial solutions. Moreover, depending on the coupling parameter, this semi-trivial solution will be a strict local minimum or a saddle point. Furthermore we show the existence of a global minimum on the Nehari manifold with energy below the energy of the semi-trivial solution, for some values of the coupling parameter. In addition, by applying the mountain-pass theorem, we find another critical point for certain values of the parameters. All of this is obtained constraining the functionals to the appropriate Nehari manifolds and, in the high-dimensional case, restricted to radial framework. This analysis is supported by some numerical evidence finding the profiles of some solutions. Published by AIP Publishing. https://doi.org/10.1063/1.5010682
\end{abstract}

\section{INTRODUCTION}

In the present work, we analyze the existence of solutions of the following higher order system, coupling nonlinear Schrödinger-Korteweg-de Vries equations

$$
\left\{\begin{aligned}
i f_{t}-f_{x x x x}+|f|^{2} f+\beta f g & =0, \\
g_{t}-g_{x x x x x}+\frac{1}{2}(|g| g)_{x}+\frac{1}{2} \beta\left(|f|^{2}\right)_{x} & =0,
\end{aligned}\right.
$$

with $f=f(x, t) \in \mathbb{C}, g=g(x, t) \in \mathbb{R}$, and $\beta \in \mathbb{R}$ the coupling parameter. This system shows phenomena of interactions between short and long dispersive waves, where $f$ represents the short-wave, while $g$ stands for the long-wave. This kind of phenomenon has been usually analyzed looking for traveling waves. However, looking for "standing-traveling" (this is the first time, to the best of our knowledge, that the interaction of standing and traveling waves has been analyzed in the mathematical literature) wave solutions of the form

$$
(f(x, t), g(x, t))=\left(e^{i \lambda_{1} t} u(x), v\left(x-\lambda_{2} t\right)\right), \quad \text { where } u, v \text { are real functions, }
$$

we arrive at the fourth-order stationary system

$$
\left\{\begin{array}{l}
u^{(4)}+\lambda_{1} u=u^{3}+\beta u v, \\
v^{(4)}+\lambda_{2} v=\frac{1}{2}|v| v+\frac{1}{2} \beta u^{2},
\end{array}\right.
$$

\footnotetext{
a) Electronic addresses: pacaudev@math.uc3m.es and pablo.caudevilla@icmat.es

b) Electronic addresses: ecolorad@math.uc3m.es and eduardo.colorado@icmat.es

c)E-mail: rfabelo@math.uc3m.es
} 
where $w^{(4)}$ denotes the fourth derivative of $w$. Although system (1) only makes sense in dimension 1 , passing to the stationary system (2), it makes sense to consider it in higher dimensional cases, as follows:

$$
\left\{\begin{array}{l}
\Delta^{2} u+\lambda_{1} u=u^{3}+\beta u v, \\
\Delta^{2} v+\lambda_{2} v=\frac{1}{2}|v| v+\frac{1}{2} \beta u^{2},
\end{array}\right.
$$

where $u, v \in W^{2,2}\left(\mathbb{R}^{N}\right), 1 \leq N \leq 7, \lambda_{j}>0$ with $j=1,2$ and $\beta>0$ is again the coupling parameter.

Higher order nonlinear Schrödinger equations have been under scrutiny during the last decades by several authors such as Fibich et al. ${ }^{18}$ Karpman and Shagalov, ${ }^{22}$ Pausader, ${ }^{26}$ or Guo, Ablowitz, and Zhu, among others. From an application point of view, we should point out that this kind of fourth order equation appears in the propagation of intense laser beams in a bulk medium with Kerr nonlinearity and have been analyzed mathematically considering this framework by Fibich et al. ${ }^{18}$ (faster transmission in fiber optics) with the purpose of obtaining stability results of solitons in magnetic materials. Note that the stability of the solitons coming from Nonlinear Schrödinger equations with power nonlinearity will depend on the dimension. Moreover, as discussed by several authors, and in relation to the problem presented here, it is important to note that the effect of the higher order terms plays a relevant role as well; see Ref. 22 for further details.

On the other hand, it is already well known that the third order KdV equation describes the evolution of weakly nonlinear and weakly dispersive shallow waves in physical contexts such as plasma, ion-acoustic waves, stratified internal, and atmospheric waves. For this third order model, much has been analyzed and obtained during the last decades. However, although it seems that the fifth order has been forgotten or never analyzed before, we must mention a not very widely known work of Baker ${ }^{8}$ and published in 1903. In particular, a fifth order KdV, which might occur for nonlinear and weakly dispersive long waves where the surface tension is large, was studied. Also, this fifth order equation might also appear for weak amplitude ionacoustic waves in unmagnetized plasma; see Ref. 20 for a long discussion about these kinds of works.

In most of those works mentioned above the analysis focuses on considering traveling waves specifically. However, in this paper for the proposed model (1), we will put under mathematical analysis and for the first time, the possible interaction between standing and traveling waves enriching the already existing family of solutions in water wave theory, after coupling a fourth-order Schrödinger equation and a $\mathrm{KdV}$ equation of fifth order.

Recently, other similar fourth-order systems studying the interaction of coupled nonlinear Schrödinger equations have appeared; see Ref. 3, where the coupling terms have the same homogeneity as the nonlinear terms. Note that, as far as we know there is not any previous mathematical work analyzing a higher order system with the nonlinear and coupling terms as the ones considered here in system (3).

Thus, in particular we first analyze the existence of solutions for system (3) in the dimensional case $2 \leq N \leq 7$ in the radial framework (see Subsection IV A) by using the compactness described in Remark 2-(iii). On the other hand, the one dimensional case is studied in Subsection IV B where we use a measure lemma from the work of Lions ${ }^{25}$ to circumvent the lack of compactness.

Thanks to the structure of system (3), the corresponding energy functional is not bounded below. For this particular reason, we constrain the problem on the Nehari manifold, having then a functional bounded below. We shall show in Sec. II, this Nehari manifold is actually a natural constraint.

Furthermore, we show that system (3) has a semi-trivial solution, $\mathbf{v}_{2}=\left(0, V_{2}\right)$ defined in Sec. III. In fact, we prove that depending on the coupling parameter, this semi-trivial solution will be a strict local minimum or a saddle point of the energy functional constrained to the Nehari manifold; see details below. Then, in order to find bound or ground state solutions, we need to check that they are different from $\mathbf{v}_{2}$, with both components different from zero.

To be more precise, we prove that there exist two threshold values of the coupling parameter $\beta$, denoted by $\Lambda^{+}$and $\Lambda^{-}$, and defined by (21) such that the associated functional constrained to the 
corresponding Nehari manifold possesses a global minimum, which is a critical point with energy below the energy of the semi-trivial solution under the following assumptions:

- either $\beta>\Lambda^{+}$,

- $\beta<\Lambda^{-}$,

- or $\Lambda^{-}<\beta<\Lambda^{+}$and $\lambda_{2} \gg 1$.

We would like to point out that one cannot follow and use Steiner symmetrization to show that any solution of (3), analyzed in $\mathbb{E}=W^{2,2}\left(\mathbb{R}^{N}\right) \times W^{2,2}\left(\mathbb{R}^{N}\right)$, is going to be radially symmetric. This fact and the compactness argument above are the main reasons to work in the radial space $\mathbb{H}=H$ $\times H$, with $H$ defined as the radially symmetric functional subspace of $W^{2,2}\left(\mathbb{R}^{N}\right)$.

Recently in Refs. 12 and 13, a similar system to (1) has been analyzed

$$
\left\{\begin{aligned}
i f_{t}+f_{x x}+|f|^{2} f+\beta f g & =0, \\
g_{t}+g_{x x x}+g g_{x}+\frac{1}{2} \beta\left(|f|^{2}\right)_{x} & =0,
\end{aligned}\right.
$$

arising in fluid mechanics, such as the interactions of capillary-gravity water waves, ${ }^{23}$ magnetized plasma, ${ }^{21,27}$ and in many physical phenomena as well, such as Bose-Einstein condensates; ${ }^{9,11}$ see Refs. 2, 12-16, and 19 for further details on similar systems.

The solutions studied in papers ${ }^{12,13}$ (see also Refs. 15 and 16) are taken as solitary traveling waves, i.e.,

$$
(f(x, t), g(x, t))=\left(e^{i \omega t} e^{i \frac{c}{2} x} u(x-c t), v(x-c t)\right), \quad \text { where } u, v \text { are real functions. }
$$

Choosing $\lambda_{1}=\omega+\frac{c^{2}}{4}$ and $\lambda_{2}=c$, then $u$ and $v$ are solutions of the following stationary system:

$$
\left\{\begin{array}{l}
-u^{\prime \prime}+\lambda_{1} u=u^{3}+\beta u v, \\
-v^{\prime \prime}+\lambda_{2} v=\frac{1}{2} v^{2}+\frac{1}{2} \beta u^{2} .
\end{array}\right.
$$

We have organized the paper as follows. In Sec. II, we introduce the notation, establish the functional framework, define the Nehari manifold and study its properties. In Sec. III, we construct a semi-trivial solution and show its geometry depending on the coupling parameter. Section IV is devoted to proving the main results of the paper. It is divided into two subsections: in the first one (Subsection IV A), we study the high-dimensional case $(2 \leq N \leq 7)$, while the second one (Subsection IV B) deals with the one-dimensional case. Finally in Sec. V, we perform a numerical analysis using a WKBJ-type asymptotic approximation and a standard shooting strategy from infinity showing the profiles of some solutions.

\section{FUNCTIONAL SETTING, NOTATION, AND NEHARI MANIFOLD}

Let $E$ be the Sobolev space $W^{2,2}\left(\mathbb{R}^{N}\right)$ then, we define the following equivalent norms and scalar products:

$$
\langle u, v\rangle_{j}:=\int_{\mathbb{R}^{N}} \Delta u \cdot \Delta v d x+\lambda_{j} \int_{\mathbb{R}^{N}} u v d x, \quad\|u\|_{j}^{2}:=\langle u, u\rangle_{j} \quad j=1,2 .
$$

Let us define the product Sobolev space $\mathbb{E}:=E \times E$ and denote its elements by $\mathbf{u}=(u, v)$ with $\mathbf{0}=(0$, $0)$. We will take the inner product in $\mathbb{E}$ as follows:

$$
\left\langle\mathbf{u}_{1}, \mathbf{u}_{2}\right\rangle:=\left\langle u_{1}, u_{2}\right\rangle_{1}+\left\langle v_{1}, v_{2}\right\rangle_{2},
$$

which induces the following norm:

$$
\|\mathbf{u}\|:=\sqrt{\|u\|_{1}^{2}+\|v\|_{2}^{2}} .
$$

We denote by $H$ the space of radially symmetric functions in $E$, and $\mathbb{H}:=H \times H$. In addition, we define the energy functional associated with system (3) by

$$
\Phi(\mathbf{u})=I_{1}(u)+I_{2}(v)-\frac{1}{2} \beta \int_{\mathbb{R}^{N}} u^{2} v d x, \quad \mathbf{u}=(u, v) \in \mathbb{E},
$$


where

$$
I_{1}(u)=\frac{1}{2}\|u\|_{1}^{2}-\frac{1}{4} \int_{\mathbb{R}^{N}} u^{4} d x, \quad I_{2}(v)=\frac{1}{2}\|v\|_{2}^{2}-\frac{1}{6} \int_{\mathbb{R}^{N}}|v|^{3} d x, \quad u, v \in E,
$$

are the energy functionals associated with the uncoupled equations in (3). We define

$$
\Psi(\mathbf{u})=\Phi^{\prime}(\mathbf{u})[\mathbf{u}]=\|\mathbf{u}\|^{2}-\int_{\mathbb{R}^{N}} u^{4} d x-\frac{1}{2} \int_{\mathbb{R}^{N}}|v|^{3} d x-\frac{3}{2} \beta \int_{\mathbb{R}^{N}} u^{2} v d x .
$$

Using the previous definition, the Nehari manifold is given by

$$
\mathcal{M}=\{\mathbf{u} \in \mathbb{E} \backslash\{\mathbf{0}\}: \Psi(\mathbf{u})=0\} .
$$

This manifold will be used in order to deal with the one dimensional case in Subsection IV B, in which there is no compactness, see Remark 2-(iii).

In the dimensional case $2 \leq N \leq 7$, we restrict the Nehari Manifold to the radial setting, denoting it as

$$
\mathcal{N}=\{\mathbf{u} \in \mathbb{H} \backslash\{\mathbf{0}\}: \Psi(\mathbf{u})=0\} .
$$

Remark 1. All the properties we are going to prove in this section are satisfied for both $\mathcal{M}$ and $\mathcal{N}$, but the Palais-Smale (PS) condition, in Lemma 3, is only satisfied for $\Phi$ on $\mathcal{N}$ because we are analyzing this problem on the radial setting, see again Remark 2-(iii).

Now, we are going to prove some properties for the Nehari manifold $\mathcal{N}$ which can be extended to the other Nehari manifold $\mathcal{M}$. Thus, differentiating expression (6) yields

$$
\Psi^{\prime}(\mathbf{u})[\mathbf{u}]=2\|\mathbf{u}\|^{2}-4 \int_{\mathbb{R}^{N}} u^{4} d x-\frac{3}{2} \int_{\mathbb{R}^{N}}|v|^{3} d x-\frac{9}{2} \beta \int_{\mathbb{R}^{N}} u^{2} v d x,
$$

and using the fact that $\Psi(\mathbf{u})=0$ for any $\mathbf{u} \in \mathcal{N}$, we have

$$
\Psi^{\prime}(\mathbf{u})[\mathbf{u}]=\Psi^{\prime}(\mathbf{u})[\mathbf{u}]-3 \Psi(\mathbf{u})=-\|\mathbf{u}\|^{2}-\int_{\mathbb{R}^{N}} u^{4} d x<0, \quad \forall \mathbf{u} \in \mathcal{N} .
$$

Then, $\mathcal{N}$ is a locally smooth manifold near any point $\mathbf{u} \neq 0$ with $\Psi(\mathbf{u})=0$. Taking the derivative of the functional $\Phi$ in the direction $\mathbf{h}=\left(h_{1}, h_{2}\right)$, we find

$$
\Phi^{\prime}(\mathbf{u})[\mathbf{h}]=I_{1}^{\prime}(u)\left[h_{1}\right]+I_{2}^{\prime}(v)\left[h_{2}\right]-\beta \int_{\mathbb{R}^{N}} u v h_{1} d x-\frac{1}{2} \beta \int_{\mathbb{R}^{N}} u^{2} h_{2} d x .
$$

The second derivative of $\Phi$ is given by

It satisfies

$$
\Phi^{\prime \prime}(\mathbf{u})[\mathbf{h}]^{2}=\|\mathbf{h}\|^{2}-3 \int_{\mathbb{R}^{N}} u^{2} h_{1}^{2} d x-\int_{\mathbb{R}^{N}}|v| h_{2}^{2} d x-\beta \int_{\mathbb{R}^{N}} v h_{1}^{2} d x-2 \beta \int_{\mathbb{R}^{N}} u h_{1} h_{2} d x .
$$

$$
\Phi^{\prime \prime}(\mathbf{0})[\mathbf{h}]^{2}=\|\mathbf{h}\|^{2},
$$

which is positive definite so that $\mathbf{0}$ is a strict minimum critical point for $\Phi$. As a consequence, we have that $\mathcal{N}$ is a smooth complete manifold, and there exists a constant $\rho>0$ such that

$$
\|\mathbf{u}\|^{2}>\rho \quad \forall \mathbf{u} \in \mathcal{N} .
$$

Notice that having (10) and (11) (Ref. 6, Proposition 6.7) (see also Ref. 5) proves that $\mathcal{N}$ is a natural constraint of $\Phi$, i.e., $\mathbf{u} \in \mathbb{H} \backslash\{\mathbf{0}\}$ is a critical point of $\Phi$ if and only if $\mathbf{u}$ is a critical point of $\Phi$ constrained on $\mathcal{N}$.

Remarks 2.

(i) The functional constrained on $\mathcal{N}$ takes the form

$$
\left.\Phi\right|_{\mathcal{N}}(\mathbf{u})=\frac{1}{6}\|\mathbf{u}\|^{2}+\frac{1}{12} \int_{\mathbb{R}^{N}} u^{4} d x .
$$

Even more, using (11) and (12),

$$
\Phi(\mathbf{u})>\frac{1}{6} \rho \quad \forall \mathbf{u} \in \mathcal{N} .
$$

Therefore, $\Phi$ is bounded from below on $\mathcal{N}$, so we can try to minimize it on the Nehari manifold. 
(ii) For $\mathbf{u} \in \mathbb{E}$, there exists a unique positive $t \in \mathbb{R}$ such that $t \mathbf{u} \in \mathcal{M}$. Note that $t$ comes from the following expression:

$$
\|\mathbf{u}\|^{2}=t^{2} \int_{\mathbb{R}^{N}} u^{4} d x+t\left(\frac{1}{2} \int_{\mathbb{R}^{N}}|v|^{3} d x+\frac{3}{2} \beta \int_{\mathbb{R}^{N}} u^{2} v d x\right) .
$$

Thus in particular if $\mathbf{u}_{1}, \mathbf{u}_{2} \in \mathcal{M}$, then the set

$$
\left\{t \mathbf{u}:=t\left[\alpha \mathbf{u}_{1}+(1-\alpha) \mathbf{u}_{2}\right]: 0 \leq \alpha \leq 1 \text {, and } t \text { is defined by }(14)\right\} \subset \mathcal{M},
$$

proving that $\mathcal{M}$ (as well as $\mathcal{N}$ ) is path-connected.

(iii) Let us define

$$
2^{*}=\left\{\begin{array}{cll}
\frac{2 N}{N-4} & \text { if } & N>4, \\
\infty & \text { if } & 1 \leq N \leq 4 .
\end{array}\right.
$$

One has the following Sobolev embedding:

$$
E \hookrightarrow L^{p}\left(\mathbb{R}^{N}\right), \quad \text { for }\left\{\begin{array}{l}
2 \leq p \leq 2^{*}, \text { if } N \neq 4, \\
2 \leq p<2^{*}, \text { if } N=4,
\end{array}\right.
$$

see, for instance, Refs. 1 and 24. In particular, these embeddings show that the functional $\Phi$ is well defined for every $1 \leq N \leq 7$. Concerning the Palais-Smale condition for $2 \leq N \leq 7$ (see Lemma 3), we will use that if $N \geq 2$, replacing $E$ by the radial subspace $H$, we have the following compact embedding:

$$
H \hookrightarrow \hookrightarrow L^{p}\left(\mathbb{R}^{N}\right), \quad \text { for } 2<p<2^{*} .
$$

The one dimensional case $(N=1)$ is analyzed in a different manner in Subsection IV B because of the lack of compactness.

To conclude this section, we also prove that the functional $\Phi$ satisfies the PS condition constrained to $\mathcal{N}$ on the high-dimensional case.

Lemma 3. Assume that $2 \leq N \leq 7$, then $\Phi$ satisfies the PS condition constrained on $\mathcal{N}$.

Proof. Let $\mathbf{u}_{n}=\left(u_{n}, v_{n}\right) \in \mathcal{N}$ be a PS sequence, i.e.,

$$
\Phi\left(\mathbf{u}_{n}\right) \rightarrow c \quad \text { and } \quad \nabla_{\mathcal{N}} \Phi\left(\mathbf{u}_{n}\right) \rightarrow 0, \quad \text { as } \quad n \rightarrow \infty .
$$

From (12) and the first convergence in (15), it follows that $\mathbf{u}_{n}$ is bounded, then we have a weakly convergent subsequence (denoted again by $\mathbf{u}_{n}$ ) $\mathbf{u}_{n} \rightarrow \mathbf{u}_{0} \in \mathbb{H}$. Since $H$ is compactly embedded into $L^{p}\left(\mathbb{R}^{N}\right)$ for $2<p<4+\frac{2}{3}$ and $2 \leq N \leq 7$ [see Remark 2-(iii)], we infer that

$$
\int_{\mathbb{R}^{N}} u_{n}^{4} d x \rightarrow \int_{\mathbb{R}^{N}} u_{0}^{4} d x, \quad \int_{\mathbb{R}^{N}}\left|v_{n}\right|^{3} d x \rightarrow \int_{\mathbb{R}^{N}}\left|v_{0}\right|^{3} d x, \quad \int_{\mathbb{R}^{N}} u_{n}^{2} v_{n} d x \rightarrow \int_{\mathbb{R}^{N}} u_{0}^{2} v_{0} d x .
$$

Moreover, using the fact that $\mathbf{u}_{n} \in \mathcal{N}$ and (11), we have

$$
\left\|\mathbf{u}_{n}\right\|^{2}=\int_{\mathbb{R}^{N}} u_{n}^{4} d x+\frac{1}{2} \int_{\mathbb{R}^{N}}\left|v_{n}\right|^{3} d x+\frac{3}{2} \beta \int_{\mathbb{R}^{N}} u_{n}^{2} v_{n} d x \rightarrow \int_{\mathbb{R}} u_{0}^{4} d x+\frac{1}{2} \int_{\mathbb{R}^{N}}\left|v_{0}\right|^{3} d x+\frac{3}{2} \beta \int_{\mathbb{R}^{N}} u_{0}^{2} v_{0} d x \geq \rho,
$$

which implies that $\mathbf{u}_{0} \neq \mathbf{0}$. The constrained gradient satisfies

$$
\nabla_{\mathcal{N}} \Phi\left(\mathbf{u}_{n}\right)=\Phi^{\prime}\left(\mathbf{u}_{n}\right)-\lambda_{n} \Psi^{\prime}\left(\mathbf{u}_{n}\right) \rightarrow 0,
$$

then, taking into account (10) and (11) the fact that $\Phi^{\prime}\left(\mathbf{u}_{n}\right)\left[\mathbf{u}_{n}\right]=\Psi\left(\mathbf{u}_{n}\right)=0$ and evaluating the identity of expression (16) at $\mathbf{u}_{n}$, we deduce that $\lambda_{n} \rightarrow 0$ as $n \rightarrow \infty$. We also have that $\left\|\Psi^{\prime}\left(\mathbf{u}_{n}\right)\right\|$ is bounded. Hence, from (16), jointly with the fact $\lambda_{n} \rightarrow 0$, we obtain

$$
\left\|\Phi^{\prime}\left(\mathbf{u}_{n}\right)\right\| \leq\left\|\nabla_{\mathcal{N}} \Phi\left(\mathbf{u}_{n}\right)\right\|+\left|\lambda_{n}\right|\left\|\Psi^{\prime}\left(\mathbf{u}_{n}\right)\right\| \rightarrow 0 \quad \text { as } \quad n \rightarrow \infty .
$$

To finish the proof since $\Phi^{\prime}\left(\mathbf{u}_{n}\right)\left[\mathbf{u}_{0}\right] \rightarrow 0$ as $n \rightarrow \infty$, it follows that $\mathbf{u}_{n} \rightarrow \mathbf{u}_{0}$ strongly. 


\section{EXISTENCE AND PROPERTIES OF SEMI-TRIVIAL SOLUTIONS}

System (3) only admits one kind of semi-trivial solution of the form $(0, v)$. Indeed, if we suppose $v=0$, the second equation in (3) gives us that $u=0$ as well. Thus, let us take $\mathbf{v}_{2}=\left(0, V_{2}\right)$, where $V_{2}$ is a radially symmetric ground state solution of the equation $\Delta^{2} v+\lambda_{2} v=\frac{1}{2}|v| v$ in $\mathbb{H}$ (not necessarily a ground state in $\mathbb{E}$ ). Moreover, if we denote by $V$ a radially symmetric ground state solution of the equation

$$
\Delta^{2} v+v=\frac{1}{2}|v| v,
$$

then, after some scaling, $V_{2}$ can be defined by

$$
V_{2}(x)=\lambda_{2} V\left(\sqrt[4]{\lambda_{2}} x\right)
$$

As a consequence, the ground state $V_{2}$ provides us with a semi-trivial solution $\mathbf{v}_{2}=\left(0, V_{2}\right)$ which actually is a semi-trivial solution of (3) with lowest energy among all the semi-trivial solutions.

We define the Nehari manifold corresponding to the second single equation of (3) by

$$
\mathcal{N}_{2}=\left\{v \in H \backslash\{0\}: J_{2}(v)=0\right\},
$$

where

$$
J_{2}(u):=I_{2}^{\prime}(u)[u] .
$$

Let us define the tangent space to $\mathcal{N}$ on $\mathbf{v}_{2}$ by

$$
T_{\mathbf{v}_{2}} \mathcal{N}:=\left\{\mathbf{h} \in \mathbb{E}: \Psi^{\prime}\left(\mathbf{v}_{2}\right)[\mathbf{h}]=0\right\},
$$

and equivalently we define the tangent space to $\mathcal{N}_{2}$ on $V_{2}$ by

$$
T_{V_{2}} \mathcal{N}_{2}:=\left\{h \in E: J_{2}^{\prime}\left(V_{2}\right)[h]=0\right\} .
$$

We can see that the following equivalence holds:

$$
\mathbf{h}=\left(h_{1}, h_{2}\right) \in T_{\mathbf{v}_{2}} \mathcal{N} \Longleftrightarrow h_{2} \in T_{V_{2}} \mathcal{N}_{2},
$$

in fact,

$$
\begin{aligned}
\mathbf{h} \in T_{\mathbf{v}_{2}} \mathcal{N} & \Longleftrightarrow \Psi^{\prime}\left(\mathbf{v}_{2}\right)[\mathbf{h}]=0 \\
& \Longleftrightarrow 2\left\langle V_{2}, h_{2}\right\rangle_{2}-\frac{3}{2} \int_{\mathbb{R}^{N}} V_{2}^{2} h_{2} d x=0 \\
& \Longleftrightarrow J_{2}^{\prime}\left(V_{2}\right)\left[h_{2}\right]=0 \\
& \Longleftrightarrow h_{2} \in T_{V_{2}} \mathcal{N}_{2} .
\end{aligned}
$$

If we denote by $D^{2} \Phi_{\mathcal{N}}$ the second derivative of $\Phi$ constrained on $\mathcal{N}$, using that $\mathbf{v}_{2}$ is a critical point of $\Phi$, plainly we obtain that

$$
D^{2} \Phi_{\mathcal{N}}\left(\mathbf{v}_{2}\right)[\mathbf{h}]^{2}=\Phi^{\prime \prime}\left(\mathbf{v}_{2}\right)[\mathbf{h}]^{2} \quad \forall \mathbf{h} \in T_{\mathbf{v}_{2}} \mathcal{N} .
$$

In the following result, we establish the character of $\mathbf{v}_{2}$ in terms of the size of the coupling parameter.

Definition 4. We define

$$
\Lambda^{+}:=\inf _{\substack{\left.\varphi \in H \\ \varphi\right|_{A} \neq 0}} \frac{\|\varphi\|_{1}^{2}}{\int_{\mathbb{R}^{n}} V_{2}^{+} \varphi^{2} d x}, \quad \text { and } \quad \Lambda^{-}:=-\inf _{\substack{\left.\varphi \in H \\ \varphi\right|_{B} \neq 0}} \frac{\|\varphi\|_{1}^{2}}{\int_{\mathbb{R}^{n}} V_{2}^{-} \varphi^{2} d x},
$$

where

$$
V_{2}^{+}(x)=\max \left\{0, V_{2}(x)\right\}, A=\operatorname{supp}\left(V_{2}^{+}\right), \quad V_{2}^{-}(x)=\max \left\{0,-V_{2}(x)\right\}, B=\operatorname{supp}\left(V_{2}^{-}\right),
$$

with $V_{2}=V_{2}^{+}-V_{2}^{-}$.

Remark 5. $V_{2}$ is an oscillatory solution to (17) of changing sign with a sequence of zeros which converges to infinity. The existence of a positive ground state solution to (17), up to our knowledge, is still unknown. See Ref. 18 for further details.

Proposition 6.

(i) If $\Lambda^{-}<\beta<\Lambda^{+}$, then $\mathbf{v}_{2}$ is a strict local minimum of $\Phi$ constrained on $\mathcal{N}$. 
(ii) If either $\beta<\Lambda^{-}$or $\beta>\Lambda^{+}$, then $\mathbf{v}_{2}$ is a saddle point of $\Phi$ constrained on $\mathcal{N}$. Moreover,

$$
\inf _{\mathcal{N}} \Phi<\Phi\left(\mathbf{v}_{2}\right)
$$

Proof.

(i) Case $\left(0 \leq \beta<\Lambda^{+}\right)$

For $\mathbf{h} \in T_{\mathbf{v}_{2}} \mathcal{N}$, one has that

or equivalently

$$
D^{2} \Phi_{\mathcal{N}}\left(\mathbf{v}_{2}\right)[\mathbf{h}]^{2}=\Phi^{\prime \prime}\left(\mathbf{v}_{2}\right)[\mathbf{h}]^{2}=\left\|h_{1}\right\|_{1}^{2}+I_{2}^{\prime \prime}\left(V_{2}\right)\left[h_{2}\right]^{2}-\beta \int_{\mathbb{R}^{N}} V_{2} h_{1}^{2} d x
$$

$$
D^{2} \Phi_{\mathcal{N}}\left(\mathbf{v}_{2}\right)[\mathbf{h}]^{2}=\left\|h_{1}\right\|_{1}^{2}+I_{2}^{\prime \prime}\left(V_{2}\right)\left[h_{2}\right]^{2}-\beta \int_{\mathbb{R}^{n}} V_{2}^{+} h_{1}^{2} d x+\beta \int_{\mathbb{R}^{n}} V_{2}^{-} h_{1}^{2} d x .
$$

First, since $0 \leq \beta<\Lambda^{+}$and the definition of $\Lambda^{+}$by (21), there exists $c_{1}>0$ such that

$$
\left\|h_{1}\right\|_{1}^{2}-\beta \int_{\mathbb{R}^{n}} V_{2}^{+} h_{1}^{2} d x \geq c_{1}\left\|h_{1}\right\|_{1}^{2}, \quad \text { for example } \quad c_{1}=1-\frac{\beta}{\Lambda^{+}} .
$$

On the other hand, using (19) and the fact that $V_{2}$ is a minimum of $I_{2}$ on $\mathcal{N}_{2}$, there exists a constant $c_{2}>0$ such that

$$
I_{2}^{\prime \prime}\left(V_{2}\right)\left[h_{2}\right]^{2} \geq c_{2}\left\|h_{2}\right\|_{2}^{2}
$$

Since

$$
\beta \int_{\mathbb{R}^{n}} V_{2}^{-} h_{1}^{2} d x \geq 0
$$

we obtain

$$
D^{2} \Phi_{\mathcal{N}}\left(\mathbf{v}_{2}\right)[\mathbf{h}]^{2} \geq c_{1}\left\|h_{1}\right\|_{1}^{2}+c_{2}\left\|h_{2}\right\|_{2}^{2},
$$

proving that $\mathbf{v}_{2}$ is a strict local minimum of $\Phi$ on $\mathcal{N}$.

Case $\left(\Lambda^{-}<\beta<0\right)$

Due to the definition of $\Lambda^{-}$by (21), it follows that there exists $c_{1}^{\prime}>0$ such that

$$
\left\|h_{1}\right\|_{1}^{2}+\beta \int_{\mathbb{R}^{n}} V_{2}^{-} h_{1}^{2} d x \geq c_{1}^{\prime}\left\|h_{1}\right\|_{1}^{2}, \quad \text { for example, } \quad c_{1}^{\prime}=1-\frac{\beta}{\Lambda^{-}} .
$$

Using (24) we can conclude analogously that $\mathbf{v}_{2}$ is a strict local minimum of $\Phi$ on $\mathcal{N}$ since

$$
-\beta \int_{\mathbb{R}^{n}} V_{2}^{+} h_{1}^{2} d x \geq 0
$$

(ii) Case $\left(\beta>\Lambda^{+}\right)$

In this case, we choose an element $\widetilde{h}_{1} \in H$ such that $\widetilde{h}_{1}=0$ in the support of $V_{2}^{+}$and

$$
\Lambda^{+}<\frac{\left\|\widetilde{h}_{1}\right\|_{1}^{2}}{\int_{\mathbb{R}^{N}} V_{2}^{+} \widetilde{h}_{1}^{2} d x}<\beta .
$$

Then, taking $\widetilde{\mathbf{h}}_{1}=\left(\widetilde{h}_{1}, 0\right) \in T_{\mathbf{v}_{2}} \mathcal{N}$, it yields

$$
D^{2} \Phi_{\mathcal{N}}\left(\mathbf{v}_{2}\right)\left[\widetilde{\mathbf{h}}_{1}\right]^{2}=\left\|\widetilde{h}_{1}\right\|_{1}^{2}-\beta \int_{\mathbb{R}^{N}} V_{2}^{+} \widetilde{h}_{1}^{2} d x<0,
$$

and taking $\widetilde{h}_{2} \in T_{V_{2}} \mathcal{N}_{2}$ different from zero, then $\widetilde{\mathbf{h}}_{2}=\left(0, \widetilde{h}_{2}\right) \in T_{\mathbf{v}_{2}} \mathcal{N}$ and

$$
D^{2} \Phi_{\mathcal{N}}\left(\mathbf{v}_{2}\right)\left[\widetilde{\mathbf{h}}_{2}\right]^{2}=I_{2}^{\prime \prime}\left(V_{2}\right)\left[\widetilde{h}_{2}\right]^{2} \geq c_{2}\left\|\widetilde{h}_{2}\right\|_{2}^{2}>0 \text {. }
$$

Therefore, $\mathbf{v}_{2}$ is a saddle point of $\Phi$ on $\mathcal{N}$ and obviously inequality (22) holds. Case $\left(\beta<\Lambda^{-}\right)$

Similar to the previous case, we can take an element $\widehat{h}_{1} \in H$ such that $\widehat{h}_{1}=0$ in the support of $V_{2}^{-}$and

$$
\beta<-\frac{\left\|\widehat{h}_{1}\right\|_{1}^{2}}{\int_{\mathbb{R}^{N}} V_{2}^{-} \widehat{h}_{1}^{2} d x}<\Lambda^{-}
$$


Then, with $\widehat{\mathbf{h}}_{1}=\left(\widehat{h}_{1}, 0\right) \in T_{\mathbf{v}_{2}} \mathcal{N}$, we obtain

$$
D^{2} \Phi_{\mathcal{N}}\left(\mathbf{v}_{2}\right)\left[\widetilde{\mathbf{h}}_{1}\right]^{2}=\left\|\widetilde{h}_{1}\right\|_{1}^{2}+\beta \int_{\mathbb{R}^{N}} V_{2}^{-} \widetilde{h}_{1}^{2} d x<0 .
$$

Therefore as in the previous case, $\mathbf{v}_{2}$ is a saddle point of $\Phi$ on $\mathcal{N}$ and (22) holds.

\section{EXISTENCE RESULTS OF NON-TRIVIAL SOLUTIONS}

This section is divided into two subsections depending on the dimension of problem (3).

\section{A. High-dimensional case, $2 \leq N \leq 7$}

In this subsection, we will see that the infimum of $\Phi$ constrained on the radial Nehari manifold $\mathcal{N}$ is attained under appropriate parameter conditions. We also prove the existence of a mountain pass critical point.

Theorem 7. Suppose either $\beta<\Lambda^{-}$or $\beta>\Lambda^{+}$, and $2 \leq N \leq 7$. The infimum of $\Phi$ on $\mathcal{N}$ is attained at some $\widetilde{\mathbf{u}}$ with $\Phi(\widetilde{\mathbf{u}})<\Phi\left(\mathbf{v}_{2}\right)$ and both components $\widetilde{u}, \widetilde{v} \equiv \equiv$.

Proof. By the Ekeland's variational principle (see Ref. 17 for further details), there exists a minimizing sequence $\mathbf{u}_{n} \in \mathcal{N}$, i.e.,

$$
\Phi\left(\mathbf{u}_{n}\right) \rightarrow c:=\inf _{\mathcal{N}} \Phi \quad \text { and } \quad \nabla_{\mathcal{N}} \Phi\left(\mathbf{u}_{n}\right) \rightarrow 0 .
$$

Due to the Lemma 3, there exists $\widetilde{\mathbf{u}} \in \mathcal{N}$ such that

$$
\mathbf{u}_{n} \rightarrow \widetilde{\mathbf{u}} \text { strongly as } n \rightarrow \infty,
$$

hence, $\widetilde{\mathbf{u}}$ is a minimum point of $\Phi$ on $\mathcal{N}$. Moreover, taking into account Proposition 6-(ii), we have

$$
\Phi(\widetilde{\mathbf{u}})=c<\Phi\left(\mathbf{v}_{2}\right) \text {. }
$$

Note that the second component $\widetilde{v}$ cannot be zero because if that occurs then $\widetilde{\mathbf{u}} \equiv 0$ due to the form of the second equation of (3), and zero is not in $\mathcal{N}$. On the other hand, if we suppose that the first component $\widetilde{u} \equiv 0$, then

$$
I_{2}(\widetilde{v})=\Phi(\widetilde{\mathbf{u}})<\Phi\left(\mathbf{v}_{2}\right)=I_{2}\left(V_{2}\right),
$$

and this is a contradiction due to the fact that $V_{2}$ is a ground state of the equation $\Delta^{2} v+\lambda_{1} v=\frac{1}{2}|v| v$.

Finally we conclude that both components are non-trivial because, due to Proposition 6, we have $\Phi(\widetilde{\mathbf{u}})$, with $I_{2}\left(V_{2}\right)$ the ground state energy level of (17). More precisely, remember that if the second component $\widetilde{v}=0$ then $\widetilde{u}=0$ which is a contradiction due to $\Phi(\widetilde{\mathbf{u}})>0$. If $\widetilde{u}=0$ since $\left.\Phi(0, \widetilde{v})=I_{2} \widetilde{v}\right)$, we find a contradiction because $V_{2}$ is a ground state of the equation $\Delta^{2} v+\lambda_{2} v=\frac{1}{2}|v| v$.

Theorem 8. Assume $2 \leq N \leq 7, \beta>0$. There exists a positive constant $\Lambda_{2}$ such that, if $\lambda_{2}>\Lambda_{2}$, the functional $\Phi$ attains its infimum on $\mathcal{N}$ at some $\widehat{\mathbf{u}}$ with $\Phi(\widehat{\mathbf{u}})<\Phi\left(\mathbf{v}_{2}\right)$ and both components $\widehat{u}, \widehat{v} \not \equiv$.

Proof. Using the same argument as above in Theorem 7, we prove that the infimum is attained at some point $\widehat{\mathbf{u}} \in \mathcal{N}$, but to show that $\widehat{u}, \widehat{v} \not \equiv 0$ we need to ensure that $\Phi(\widehat{\mathbf{u}})<\Phi\left(\mathbf{v}_{2}\right)$. In Theorem 7 , this fact was proved for either $\beta<\Lambda^{-}$or $\beta>\Lambda^{+}$and here we need to prove it for $\Lambda^{-}<\beta<\Lambda^{+}$. In this case, $\mathbf{v}_{2}$ is a strict local minima of $\Phi$ on $\mathcal{N}$ but this does not guarantee that $\widehat{\mathbf{u}} \not \equiv \mathbf{v}_{2}$.

Then, to see $\Phi(\widehat{\mathbf{u}})<\Phi\left(\mathbf{v}_{2}\right)$, we will prove that there exists an element of the form

$$
\mathbf{w}=t\left(V_{2}, V_{2}\right) \in \mathcal{N} \quad \text { with } \quad \Phi(\mathbf{w})<\Phi\left(\mathbf{v}_{2}\right),
$$

for $\lambda_{2}$ large enough.

Notice that, thanks to the equation $\Psi(\mathbf{w})=0$ we have that any $t>0$ satisfies the following condition:

$$
t^{2}\left\|\left(V_{2}, V_{2}\right)\right\|^{2}-t^{4} \int_{\mathbb{R}^{N}} V_{2}^{4} d x-\frac{1}{2} t^{3}(1+3 \beta) \int_{\mathbb{R}^{N}} V_{2}^{3} d x=0
$$


and by definition we also have

$$
\left\|\left(V_{2}, V_{2}\right)\right\|^{2}=2\left\|V_{2}\right\|_{2}^{2}+\left(\lambda_{1}-\lambda_{2}\right) \int_{\mathbb{R}^{N}} V_{2}^{2} d x
$$

Moreover, since $V_{2} \in \mathcal{N}_{2}$, we have

$$
\left\|V_{2}\right\|_{2}^{2}-\frac{1}{2} \int_{\mathbb{R}^{N}} V_{2}^{3} d x=0
$$

Substituting (27) and (28) in (26), it follows

$$
t^{2}\left(\int_{\mathbb{R}^{N}} V_{2}^{3} d x+\left(\lambda_{1}-\lambda_{2}\right) \int_{\mathbb{R}^{N}} V_{2}^{2} d x\right)-t^{4} \int_{\mathbb{R}^{N}} V_{2}^{4} d x-\frac{1}{2} t^{3}(1+3 \beta) \int_{\mathbb{R}^{N}} V_{2}^{3} d x=0 .
$$

Hence, applying the scaling (18) yields

$$
\int_{\mathbb{R}^{N}} V_{2}^{p} d x=\lambda_{2}^{p-\frac{N}{4}} \int_{\mathbb{R}^{N}} V^{p} d x
$$

Subsequently, substituting (30) for $p=2,3,4$ into (29) and dividing by $t^{2} \lambda_{2}^{3-\frac{N}{4}}$, we have that

$$
\int_{\mathbb{R}^{N}} V^{3} d x+\frac{\lambda_{1}-\lambda_{2}}{\lambda_{2}} \int_{\mathbb{R}^{N}} V^{2} d x-t^{2} \lambda_{2} \int_{\mathbb{R}^{N}} V^{4} d x-\frac{1}{2} t(1+3 \beta) \int_{\mathbb{R}^{N}} V_{2}^{3} d x=0 .
$$

Moreover, from (12), (27), and (28), we find, respectively, the expressions

$$
\begin{gathered}
\Phi(\mathbf{w})=\frac{1}{6} t^{2}\left(\int_{\mathbb{R}^{N}} V_{2}^{3} d x+\left(\lambda_{1}-\lambda_{2}\right) \int_{\mathbb{R}^{N}} V_{2}^{2} d x\right)+\frac{1}{12} t^{4} \int_{\mathbb{R}^{N}} V_{2}^{4} d x, \\
\Phi\left(\mathbf{v}_{2}\right)=I_{2}\left(V_{2}\right)=\frac{1}{2}\left\|V_{2}\right\|_{2}^{2}-\frac{1}{6} \int_{\mathbb{R}^{N}} V_{2}^{3}=\frac{1}{12} \int_{\mathbb{R}^{N}} V_{2}^{3} .
\end{gathered}
$$

Furthermore, we are looking for the inequality $\Phi(\mathbf{w})<\Phi\left(\mathbf{v}_{2}\right)$, or equivalently,

$$
\frac{1}{6} t^{2}\left(\int_{\mathbb{R}^{N}} V_{2}^{3} d x+\left(\lambda_{1}-\lambda_{2}\right) \int_{\mathbb{R}^{N}} V_{2}^{2} d x\right)+\frac{1}{12} t^{4} \int_{\mathbb{R}^{N}} V_{2}^{4} d x-\frac{1}{12} \int_{\mathbb{R}^{N}} V_{2}^{3} d x<0,
$$

and then, applying again (30) and multiplying (34) by $6 \lambda^{\frac{N}{4}-3}$, we actually have

$$
t^{2}\left(\int_{\mathbb{R}^{N}} V^{3} d x+\frac{\lambda_{1}-\lambda_{2}}{\lambda_{2}} \int_{\mathbb{R}^{N}} V^{2} d x\right)+\frac{1}{2} t^{4} \lambda_{2} \int_{\mathbb{R}^{N}} V^{4} d x-\frac{1}{2} \int_{\mathbb{R}^{N}} V^{3} d x<0 .
$$

For $\lambda_{2}$ large enough, we find that (31) will provide us with (35). Therefore, there exists a positive constant $\Lambda_{2}$ such that for $\lambda_{2}>\Lambda_{2}$ inequality (35) holds, proving that

$$
\Phi(\widehat{\mathbf{u}}) \leq \Phi(\mathbf{w})<\Phi\left(\mathbf{v}_{2}\right) .
$$

Finally, to show that $\widehat{u}, \widehat{v} \not \equiv$, we can use the same argument as in Theorem 7 .

In the following, we will prove the existence of a MP critical point of $\Phi$ on $\mathcal{N}$.

Theorem 9. Assume $2 \leq N \leq 7$ and $\Lambda^{-}<\beta<\Lambda^{+}$. There exists a constant $\Lambda_{2}$ such that if $\lambda_{2}$ $>\Lambda_{2}$, then $\Phi$ constrained on $\mathcal{N}$ has a mountain-pass critical point $\mathbf{u}^{*}$ with $\Phi\left(\mathbf{u}^{*}\right)>\Phi\left(\mathbf{v}_{2}\right)$.

Proof. Due to Proposition 6-(i), $\mathbf{v}_{2}$ is a strict local minima of $\Phi$ on $\mathcal{N}$, and taking into account Theorem 8, we obtain $\Lambda_{2}$ such that for $\lambda_{2}>\Lambda$, we have $\Phi(\widehat{\mathbf{u}})<\Phi\left(\mathbf{v}_{2}\right)$. Under these conditions, we are able to apply the mountain-pass theorem (see Ref. 7 for further details) to $\Phi$ on $\mathcal{N}$ that provide us with a PS sequence $\mathbf{v}_{n} \in \mathcal{N}$ such that

$$
\Phi\left(\mathbf{v}_{n}\right) \rightarrow c:=\inf _{\gamma \in \Gamma} \max _{0 \leq t \leq 1} \Phi(\gamma(t)),
$$

where

$$
\Gamma:=\left\{\gamma:[0,1] \rightarrow \mathcal{N} \text { continuous } \mid \gamma(0)=\mathbf{v}_{2}, \gamma(1)=\widehat{\mathbf{u}}\right\}
$$


Note that $\Gamma$ is well defined because of $\mathcal{N}$ is path-connected.

Furthermore, applying Lemma 3, we are able to find a subsequence of $\mathbf{v}_{n}$ such that (relabeling) $\mathbf{v}_{n} \rightarrow \mathbf{u}^{*}$ strongly in $\mathbb{H}$. Thus, $\mathbf{u}^{*}$ is a critical point of $\Phi$ satisfying

$$
\Phi\left(\mathbf{u}^{*}\right)>\Phi\left(\mathbf{v}_{2}\right),
$$

which conclude the proof.

\section{B. One-dimensional case, $N=1$}

Here we must point out that we do not have the compact embedding even for $\mathbb{H}$. However, we will show that for a PS sequence, we are able to find a subsequence for which its weak limit is a solution of (3) belonging to $\mathbb{E}$. Thus, in order to avoid the lack of compactness for $N=1$, we will use the following result of measure theory that one can find in Ref. 25.

Lemma 10. If $2<q<\infty$, there exists a constant $C>0$ so that

$$
\int_{\mathbb{R}}|u|^{q} d x \leq C\left(\sup _{z \in \mathbb{R}} \int_{|x-z|<1}|u(x)|^{2} d x\right)^{\frac{q-2}{2}}\|u\|_{E}^{2}, \quad \forall u \in E .
$$

The next result is the analogous to Theorem 7 for the one-dimensional case but working on the full Nehari manifold $\mathcal{M}$ defined by (7).

Theorem 11. Suppose $N=1$ and either $\beta<\Lambda^{-}$or $\beta>\Lambda^{+}$. The infimum of $\Phi$ on $\mathcal{M}$ is attained at some $\overline{\mathbf{u}}$ with both components $\bar{u}, \bar{v} \not \equiv$. Moreover, $\Phi(\overline{\mathbf{u}})<\Phi\left(\mathbf{v}_{2}\right)$.

Proof. Again, by the Ekeland's variational principle, there exists a PS sequence $\mathbf{u}_{n} \in \mathcal{M}$, i.e.,

$$
\Phi\left(\mathbf{u}_{n}\right) \rightarrow c:=\inf _{\mathcal{M}} \Phi \quad \text { and } \quad \nabla_{\mathcal{M}} \Phi\left(\mathbf{u}_{n}\right) \rightarrow 0
$$

such that $\mathbf{u}_{n}$ is bounded since (12). Also, we can assume that the sequence $\mathbf{u}_{n}$ possesses a subsequence such that (relabeling) it weakly converges $\mathbf{u}_{n} \rightarrow \mathbf{u}$ in $\mathbb{E}, \mathbf{u}_{n} \rightarrow \mathbf{u}$ strongly in $\mathbb{L}_{l o c}^{q}(\mathbb{R})=L_{l o c}^{q}(\mathbb{R}) \times L_{l o c}^{q}(\mathbb{R})$ for every $1 \leq q<\infty$ and $\mathbf{u}_{k} \rightarrow \mathbf{u}$ a.e. in $\mathbb{R}$. Moreover, arguing in the same way as in Lemma 3, we obtain $\Phi^{\prime}\left(\mathbf{u}_{n}\right) \rightarrow 0$ as $n \rightarrow \infty$.

Furthermore, we will prove that there is no loss of mass at infinity for $\mu_{n}(x):=u_{n}^{2}(x)+v_{n}^{2}(x)$, where $\mathbf{u}_{n}=\left(u_{n}, v_{n}\right)$, i.e., there exist $R, C>0$ such that

$$
\sup _{z \in \mathbb{R}} \int_{|z-x|<R} \mu_{n}(x) d x \geq C>0, \quad \forall n \in \mathbb{N} .
$$

On the contrary, if we suppose

$$
\sup _{z \in \mathbb{R}} \int_{|z-x|<R} \mu_{k}(x) d x \rightarrow 0,
$$

and thanks to Lemma 10 applied in a similar way as in Ref. 10, we find that $\mathbf{u}_{k} \rightarrow \mathbf{0}$ strongly in $\mathbb{L}^{q}(\mathbb{R})$ for any $2<q<\infty$. This is a contradiction since $\mathbf{u}_{n} \in \mathcal{N}$, and from (13) jointly with the fact $\Phi\left(\mathbf{u}_{n}\right)$ $\rightarrow c$, we have

$$
0<\frac{1}{7} \rho<c+o_{n}(1)=\Phi\left(\mathbf{u}_{n}\right), \quad \text { with } o_{n}(1) \rightarrow 0 \quad \text { as } n \rightarrow \infty,
$$

hence (37) is true and there is no loss of mass at infinity.

We observe that there is a sequence of points $\left\{z_{n}\right\} \subset \mathbb{R}$ such that by (37), the translated sequence $\bar{\mu}_{n}(x)=\mu_{n}\left(x+z_{n}\right)$ satisfies

$$
\liminf _{n \rightarrow \infty} \int_{B_{R}(0)} \bar{\mu}_{n} d x \geq C>0 .
$$

Taking into account that $\bar{\mu}_{n} \rightarrow \bar{\mu}$ strongly in $L_{l o c}^{1}(\mathbb{R})$, we obtain that $\bar{\mu} \not \equiv 0$; thus, the weak limit of $\overline{\mathbf{u}}_{n}(x):=\mathbf{u}_{n}\left(x+z_{n}\right)$, which we denote it by $\overline{\mathbf{u}}$, is non-trivial. Notice that $\overline{\mathbf{u}}_{n}, \overline{\mathbf{u}} \in \mathcal{M}$ and $\overline{\mathbf{u}}_{n}$ is the $P S$ sequence of level $c$ for $\Phi$ on $\mathcal{M}$. Moreover, if we set $F=\left.\Phi\right|_{\mathcal{M}}$ [similar to (12)] and using Fatou's lemma, we obtain the following:

$$
\Phi(\overline{\mathbf{u}})=F(\overline{\mathbf{u}}) \leq \liminf _{n \rightarrow \infty} F\left(\overline{\mathbf{u}}_{n}\right)=\liminf _{n \rightarrow \infty} \Phi\left(\overline{\mathbf{u}}_{n}\right)=\liminf _{n \rightarrow \infty} \Phi\left(\mathbf{u}_{n}\right)=c .
$$


Therefore, $\overline{\mathbf{u}}$ is a non-trivial critical point of $\Phi$ constrained on $\mathcal{M}$. Furthermore, it is not a semi-trivial solution because of $\Phi(\overline{\mathbf{u}})<\Phi\left(\mathbf{v}_{2}\right)$ from Proposition 6-(ii).

Theorem 8 can by extended to the one-dimensional case directly using the same idea as we have performed in the last proof, obtaining the following.

Corollary 12. Assume $N=1, \beta>0$. There exists a positive constant $\Lambda_{2}$ such that if $\lambda_{2}>\Lambda_{2}$, the functional $\Phi$ attains its infimum on $\mathcal{N}$ at some $\widehat{\mathbf{u}}$ with $\Phi(\widehat{\mathbf{u}})<\Phi\left(\mathbf{v}_{2}\right)$ and both $\widehat{u}, \widehat{v} \equiv 0$.

To finish, for $N=1$, Theorem 9 can be obtained in a similar manner, obtaining the following.

Corollary 13. Assume $N=1$ and $\Lambda^{-}<\beta<\Lambda^{+}$. There exists a constant $\Lambda_{2}$ such that if $\lambda_{2}>\Lambda_{2}$, then $\Phi$ constrained on $\mathcal{N}$ has a mountain-pass critical point $\mathbf{u}^{*}$ with $\Phi\left(\mathbf{u}^{*}\right)>\Phi\left(\mathbf{v}_{2}\right)$.

\section{NUMERICAL ANALYSIS}

In this section, we will perform a numerical analysis of the solutions of system (3) in the radial geometry; see Ref. 4 for a similar analysis. Then, we will consider $\mathbf{u}=\mathbf{u}(r)=(u(r), v(r))$, thus, the bi-Laplacian operator takes the form

$$
\Delta^{2} u=u^{(4)}+\frac{2(N-1)}{r} u^{\prime \prime \prime}+\frac{(N-1)(N-3)}{r^{2}} u^{\prime \prime}-\frac{(N-1)(N-3)}{r^{3}} u^{\prime} .
$$

Substituting the above equation into (3), we obtain the following radial problem

$$
\left\{\begin{array}{l}
u^{(4)}+\frac{2(N-1)}{r} u^{\prime \prime \prime}+\frac{(N-1)(N-3)}{r^{2}} u^{\prime \prime}-\frac{(N-1)(N-3)}{r^{3}} u^{\prime}+\lambda_{1} u=u^{3}+\beta u v \\
v^{(4)}+\frac{2(N-1)}{r} v^{\prime \prime \prime}+\frac{(N-1)(N-3)}{r^{2}} v^{\prime \prime}-\frac{(N-1)(N-3)}{r^{3}} v^{\prime}+\lambda_{2} v=\frac{1}{2}|v| v+\frac{1}{2} \beta u^{2}
\end{array} .\right.
$$

As we have proved, the solutions of this problem vanish at infinity, and we will use a two scale WKBJtype asymptotics to approximate $\mathbf{u}(r)$ when $r$ is large enough. We assume that $\mathbf{u}$ has an exponential decay at infinity of the form

$$
\mathbf{u}(r) \approx \mathbf{u}_{\infty}(r)=\left(r^{\alpha_{1}} e^{\delta_{1} r}, r^{\alpha_{2}} e^{\delta_{2} r}\right), \quad \text { as } r \rightarrow \infty,
$$

where $\delta_{j}$ and $\alpha_{j}$ with $j=1,2$ come from, balancing, respectively, the first and second leading terms to zero on the left-hand side in (40). Indeed, substituting $\mathbf{u}_{\infty}$ into (39), the left-hand side of the first equation divided by $e^{\delta_{1} r}$ takes the form

$$
\begin{gathered}
e^{-\delta_{1} r}\left(u^{(4)}+\frac{2(N-1)}{r} u^{\prime \prime \prime}+\frac{(N-1)(N-3)}{r^{2}} u^{\prime \prime}-\frac{(N-1)(N-3)}{r^{3}} u^{\prime}+\lambda_{1} u\right)= \\
\alpha_{1}\left(\alpha_{1}-1\right)\left(\alpha_{1}-2\right)\left(\alpha_{1}-3\right) r^{\alpha_{1}-4}+4 \alpha_{1}\left(\alpha_{1}-1\right)\left(\alpha_{1}-2\right) \delta_{1} r^{\alpha_{1}-3}+6 \alpha_{1}\left(\alpha_{1}-1\right) \delta_{1}^{2} r^{\alpha_{1}-2}+4 \alpha_{1} \delta_{1}^{3} r^{\alpha_{1}-1}+ \\
\delta_{1}^{4} r^{\alpha_{1}}+2(N-1)\left[\alpha_{1}\left(\alpha_{1}-1\right)\left(\alpha_{1}-2\right) r^{\alpha_{1}-4}+3 \alpha_{1}\left(\alpha_{1}-1\right) \delta_{1} r^{\alpha_{1}-3}+3 \alpha_{1} \delta_{1}^{2} r^{\alpha_{1}-2}+\delta_{1}^{3} r^{\alpha_{1}-1}\right]+ \\
(N-1)(N-3)\left[\alpha_{1}\left(\alpha_{1}-1\right) r^{\alpha_{1}-4}+2 \alpha_{1} \delta_{1} r^{\alpha_{1}-3}+\delta_{1}^{2} r^{\alpha_{1}-2}-\alpha_{1} r^{\alpha_{1}-4}-\delta_{1} r^{\alpha_{1}-3}\right]+\lambda_{1} r^{\alpha_{1}} .
\end{gathered}
$$

Taking the first and second leading terms above, which correspond to $r_{1}^{\alpha}$ and $r^{\alpha_{1}-1}$, respectively, we arrive at the equations

$$
\delta_{1}^{4}+\lambda_{1}=0 \quad \text { and } \quad 2 \alpha_{1}+N-1=0 .
$$

Following the same procedure in the second equation of (39), we obtain similar conditions on the parameters, i.e.,

$$
\alpha_{j}=-\frac{N-1}{2} \quad \text { and } \quad \delta_{j}^{4}=-\lambda_{j} \quad \text { with } j=1,2 .
$$

Taking into account that $\mathbf{u}(r)$ vanish at infinity, we have

$$
\delta_{j}=-\frac{\sqrt[4]{\lambda_{j}}}{\sqrt{2}} \pm \frac{\sqrt[4]{\lambda_{j}}}{\sqrt{2}} i, \quad \text { with } j=1,2,
$$

and

$$
\mathbf{u}_{\infty}(r)=r^{-\frac{N-1}{2}}\left(u_{\infty}(r), v_{\infty}(r)\right),
$$


with

$$
\begin{aligned}
& u_{\infty}(r)=e^{-\frac{\sqrt[4]{\lambda_{1}}}{\sqrt{2}} r}\left(C_{11} \cos \left(\frac{\sqrt[4]{\lambda_{1}}}{\sqrt{2}} r\right)+C_{12} \sin \left(\frac{\sqrt[4]{\lambda_{1}}}{\sqrt{2}} r\right)\right), \\
& v_{\infty}(r)=e^{-\frac{\sqrt[4]{\lambda_{2}}}{\sqrt{2}} r}\left(C_{21} \cos \left(\frac{\sqrt[4]{\lambda_{2}}}{\sqrt{2}} r\right)+C_{22} \sin \left(\frac{\sqrt[4]{\lambda_{2}}}{\sqrt{2}} r\right)\right),
\end{aligned}
$$

where $C_{j i} \in \mathbb{R}, i, j=1,2$ are constants. Notice that the above expressions can be written in a more simple fashion with other constants $k_{j i} \in \mathbb{R}, i, j=1,2$ as follows:

$$
\begin{aligned}
& u_{\infty}(r)=k_{11} e^{-\frac{\sqrt[4]{\lambda_{1}}}{\sqrt{2}} r} \cos \left(\frac{\sqrt[4]{\lambda_{1}}}{\sqrt{2}}\left(r-k_{12}\right)\right) \\
& v_{\infty}(r)=k_{21} e^{-\frac{\sqrt[4]{\lambda_{2}}}{\sqrt{2}} r} \cos \left(\frac{\sqrt[4]{\lambda_{2}}}{\sqrt{2}}\left(r-k_{22}\right)\right)
\end{aligned}
$$

Once, we have this estimation of $\mathbf{u}(r)$ at infinity, we apply the standard shooting strategy from $r$ large enough, where the constants $k_{j i}$ are taken such that $\mathbf{u}(r)$ is even at the origin, i.e.,

$$
\mathbf{u}^{\prime}(0)=\mathbf{u}^{\prime \prime \prime}(0)=\mathbf{0} .
$$

Note that there are four conditions involved in the above equalities, two per each component, and we have four constants $k_{j i}$ which balance algebraically the shooting problem.

Figure 1 was generated by Matlab's solver ode $15 \mathrm{~s}$ with boundary conditions given by $\mathbf{u}_{\infty}(r)$ at $r=25$, and error tolerance $\operatorname{RelTol}=\mathrm{AbsTol}=10^{-13}$. (a) shows the solution of the system without coupling terms, while (b) shows the oscillation exponentially decaying tail. Analogously, (c) and (d) show a solution for the coupling parameter $\beta=1$.

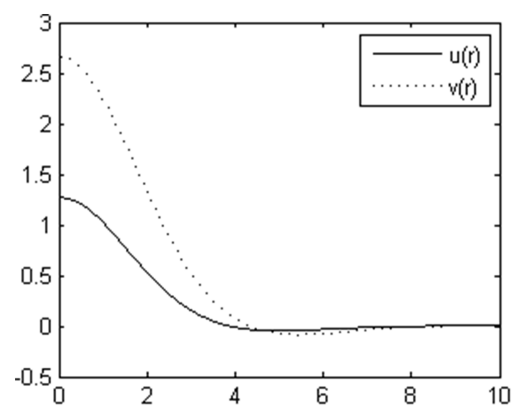

(A)

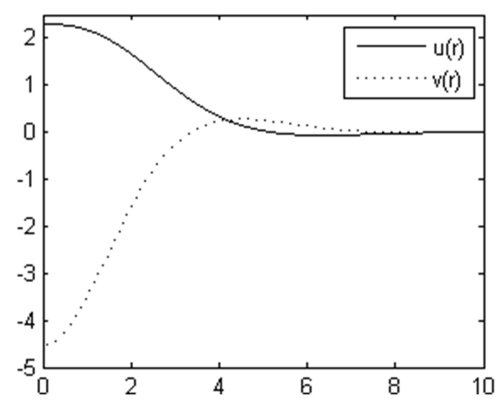

(C)

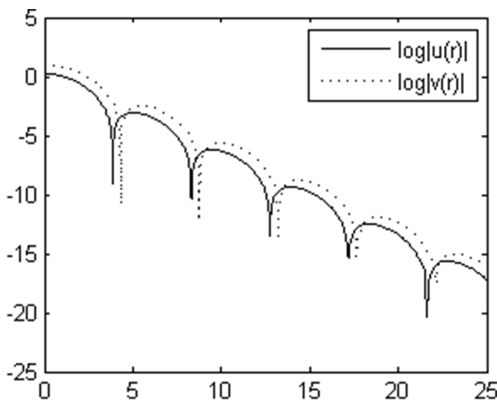

(B)

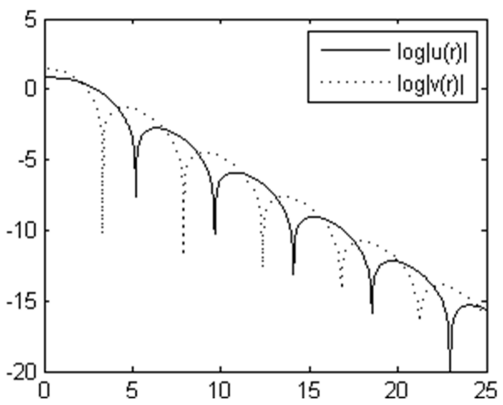

(D)

FIG. 1. Numerical solution of system (39) with $N=1$ and $\lambda_{1}=\lambda_{2}=1$. (a) and (b) correspond to $\beta=0$ and $k_{11}=2.2340$, $k_{12}=1.6390, k_{21}=-5.5528, k_{22}=6.5485$. (c) and (d) correspond to $\beta=1$ and $k_{11}=7.8306, k_{12}=2.9776, k_{21}=9.5870$, $k_{22}=5.6798$. 


\section{ACKNOWLEDGMENTS}

The authors want to thank the anonymous Referee for his valuable work that improves the final version of the paper. The first author was partially supported by the Ministry of Economy and Competitiveness of Spain under research Project No. RYC-2014-15284. The second author was partially supported by Ministry of Economy and Competitiveness of Spain and FEDER funds, under research Project No. MTM2013-44123-P.

${ }^{1}$ Adams, R. A. and Fournier, J. F., "Sobolev spaces," in Pure and Applied Mathematics, 2nd ed. (Elsevier/Academic Press, Amsterdam, 2003), Vol. 140.

${ }^{2}$ Albert, J. and Angulo Pava, J., "Existence and stability of ground-state solutions of a Schrödinger-KdV system,” Proc. R. Soc. Edinburgh, Sect. A: Math. 133, 987-1029 (2003).

3 Álvarez-Caudevilla, P., Colorado, E., and Galaktionov, V., "Existence of solutions for a system of coupled nonlinear stationary bi-harmonic Schrödinger equations," Nonlinear Anal.: Real World Appl. 23, 78-93 (2015).

${ }^{4}$ Álvarez-Caudevilla, P., Evans, J. D., and Galaktionov, V., "Countable families of solutions of a limit stationary semilinear fourth-order Cahn-Hilliard-type equation I. Mountain pass and Lusternik-Schnirel'man patterns in $\mathbb{R}^{N}$," Boundary Value Probl. 171 (2016).

${ }^{5}$ Ambrosetti, A. and Colorado, E., "Standing waves of some coupled nonlinear Schrödinger equations," J. London Math. Soc. 75(1), 67-82 (2007).

${ }^{6}$ Ambrosetti, A. and Malchiodi, A., Nonlinear Analysis and Semilinear Elliptic Problems, Volume 104 of Cambridge Studies in Advanced Mathematics (Cambridge University Press, Cambridge, 2007).

${ }^{7}$ Ambrosetti, A. and Rabinowitz, P. H., "Dual variational methods in critical point theory and applications," J. Funct. Anal. 14, 349-381 (1973).

${ }^{8}$ Baker, H. F., "On a system of differential equations leading to periodic functions," Acta Math. 27(1), 135-156 (1903).

${ }^{9}$ Chen, Z., "Solutions of nonlinear Schrödinger systems," Ph.D. dissertation (Tsinghua University, Beijing, 2014); Chen, Z., "Solutions of nonlinear Schrödinger systems," Springer thesis, Springer, Heidelberg, 2015, xii+180 pp., ISBN: 978-3-66245477-0; 978-3-662-45478-7.

${ }^{10}$ Colorado, E., "Existence results for some systems of coupled fractional nonlinear Schrödinger equations," in Recent Trends in Nonlinear Partial Differential Equations II. Stationary Problems, Volume 595 of Contemporary Mathematics (American Mathematical Society, Providence, RI, 2013), pp. 135-150.

${ }^{11}$ Colorado, E., "Positive solutions to some systems of coupled nonlinear Schrödinger equations," Nonlinear Anal. 110, 104-112 (2014)

${ }^{12}$ Colorado, E., "Existence of bound and ground states for a system of coupled nonlinear Schrödinger-KdV equations," C. R. Acad. Sci. Paris Sér. I Math. 353(6), 511-516 (2015).

${ }^{13}$ Colorado, E., "On the existence of bound and ground states for a system of coupled nonlinear Schrödinger-Korteweg-de Vries Equations," Adv. Nonlinear Anal. 6(4), 407-426 (2017).

${ }^{14}$ Corcho, A. J. and Linares, F., "Well-posedness for the Schrödinger-Korteweg-de Vries system," Trans. Am. Math. Soc. 359, 4089-4106 (2007).

${ }^{15}$ Dias, J.-P., Figueira, M., and Oliveira, F., "Existence of bound states for the coupled Schrödinger-KdV system with cubic nonlinearity," C. R. Math. Acad. Sci. Paris 348(19-20), 1079-1082 (2010).

${ }^{16}$ Dias, J.-P., Figueira, M., and Oliveira, F., "Well-posedness and existence of bound states for a coupled Schrödinger-gKdV system," Nonlinear Anal. 73(8), 2686-2698 (2010).

${ }^{17}$ Ekeland, I., "On the variational principle," J. Math. Anal. Appl. 47, 324-353 (1974).

${ }^{18}$ Fibich, G., Ilan, B., and Papanicolau, G., "Self-focusing with fourth-order dispersion,” SIAM J. Appl. Math. 62, 1437-1462 (2002).

${ }^{19}$ Funakoshi, M. and Oikawa, M., "The resonant interaction between a long internal gravity wave and a surface gravity wave packet,” J. Phys. Soc. Jpn. 52, 1982-1995 (1983).

${ }^{20}$ Galaktionov, V., Mitidieri, E., and Pohozaev, S., "Blow-up for higher-order parabolic, hyperbolic, dispersion and Schrödinger equations," in Monographs and Research Notes in Mathematics (CRC Press, Boca Raton, FL, 2015)

${ }^{21}$ Karpman, V., "On the dynamics of sonic-Langmuir solitons," Phys. Scr. 11, 263-265 (1975).

${ }^{22}$ Karpman, V. and Shagalov, A. G., "Stability of solitons described by nonlinear Schrödinger-type equations with higher order dispersion," Phys. D 144, 194-210 (2000).

${ }^{23}$ Kawahara, T., Sugimoto, N., and Kakutani, T., "Nonlinear interaction between short and long capillary-gravity waves," Stud. Appl. Math. 39, 1379-1386 (1975).

${ }^{24}$ Lions, P. L., "Symétrie et compacité dans les espaces de Sobolev," J. Funct. Anal. 49(3), 315-334 (1982).

${ }^{25}$ Lions, P. L., "The concentration-compactness principle in the calculus of variations. The locally compact case," Ann. Inst. Henri Poincare Anal. Non Linéaire 1, 223-283 (1984).

${ }^{26}$ Pausader, B., "The cubic fourth order Schrödinger equation,” J. Funct. Anal. 256, 2473-2517 (2009).

27 Yajima, N. and Oikawa, M., "Formation and interaction of sonic-Langmuir solitons: Inverse scattering method," Prog. Theor. Phys. 56, 1719-1739 (1976). 